ORIGINAL ARTICLE

\title{
Emergency department thrombolysis improves door to needle times
}

\author{
A R Corfield, C A Graham, J N Adams, I Booth, A C McGuffie
}

Emerg Med J 2004;21:676-680. doi: 10.1136/emj.2004.014449

See end of article for authors' affiliations .....................

Correspondence to: Dr C McGuffie, Accident and Emergency Department, Crosshouse Hospital, Kilmarnock $\mathrm{KA} 2 \mathrm{OBE}, \mathrm{UK}$; crawford.mcguffie@ aaaht.scot.nhs.uk

Accepted for publication 5 August 2004

\begin{abstract}
Objective: To identify the effect on door to needle (DTN) time of moving the site of thrombolysis delivery from the coronary care unit (CCU) to the emergency department (ED). To ascertain if moving the site of thrombolysis enables appropriate use of thrombolysis.

Design: Prospective cohort study.

Setting: CCU and ED of a 450 bed Scottish district general hospital without on-site primary angioplasty. Participants: Primary site for thrombolysis of patients presenting to the hospital with ST elevation MI (STEMI) moved from CCU to ED on 1 April 2000. Study patients who had a confirmed STEMI and/or received thrombolytic therapy before this date were defined as the pre-change group; those who were diagnosed as STEMI and/or received thrombolytic therapy after this date were defined as the post-change group.

Statistical analysis: Mann-Whitney test was used to compare medians and $\chi^{2}$ test for categorical data. Results: 1349 patients were discharged from CCU with a diagnosis of STEMI or received thrombolysis in the ED or CCU between April 1998 and April 2002. There were 632 patients in the pre-change group and 654 patients in the post-change group. Sixty three patients were excluded. Median DTN time for the prechange group (321 thrombolysed patients) was 64 minutes and median DTN time for the post-change group (324 thrombolysed patients) was 35 minutes, a median difference of 25 minutes $195 \% \mathrm{Cl}$ for difference 20 to 29 minutes, $p<0.0001$, Mann-Whitney $U$ test). A total of 37 patients were thrombolysed but did not have a final diagnosis of STEMI.

Conclusion: A significant reduction in DTN times accompanied this change in practice in this hospital.
\end{abstract}

M ortality and morbidity after ST elevation myocardial infarction (STEMI) has been shown to decrease after reperfusion therapy. ${ }^{1-4}$ In the United Kingdom, this is usually in the form of thrombolytic therapy rather than acute angioplasty. ${ }^{5}$ It is clear that most of the observed improvement in mortality and morbidity occurs within the first three hours after STEMI. ${ }^{6-10}$

Previous reports have considered the sources of delay in administration of thrombolysis for STEMI ${ }^{1-19}$ as measured by door to needle (DTN) time. In-hospital delays, specifically duplication of medical assessment, have been consistently identified as important causes of untimely thrombolytic therapy. ${ }^{11} 1217$

Various national bodies have repeatedly recommended time based standards for thrombolysis for STEMI based on either the initial call for help or from the time of arrival at hospital. ${ }^{28} 3233$ In England, the MINAP audit is ongoing. ${ }^{20}$

Emergency departments (EDs) in the UK, US, and Australasia have assumed a progressively greater role in the primary resuscitation and treatment of patients presenting with acute illness. Accordingly EDs have been staffed to mount a senior emergency response with most departments having 24 hour, on site, experienced middle grade or consultant cover to support junior staff.

Over this same time period, in-patient specialties have seen an inexorable rise in the emergency workload. These pressures mean that occasionally providing a senior response to emergencies can be delayed. Hospital geography can also be crucial in terms of response times.

Various solutions to this have been suggested, including the use of dedicated thrombolytic nurse specialists, ${ }^{15} 1819$ "fast track" systems to ensure rapid transfer from the ED to coronary care units (CCU), ${ }^{13}$ or direct access to a CCU. ${ }^{14}{ }^{16}$ In a number of centres this has been addressed by the decision to transfer the site of emergency thrombolysis for myocardial infarction from CCU to the ED.

Previous studies, notably from Australasia, have shown the reduction of DTN times for thrombolytic therapy when the site of thrombolysis is changed from CCU to the ED. ${ }^{21-24}$

In this hospital, until April 2000, thrombolytic therapy was routinely given in CCU. The decision to change this policy to administration of thrombolytic therapy in the ED provided a valuable opportunity to prospectively assess the effect of this change on DTN in our hospital.

The aim of this study was to prospectively assess the effect on DTN of changing the primary location for thrombolytic therapy from CCU to the ED in this Scottish district general hospital with a high incidence of ischaemic heart disease. We also aimed to assess whether ED physicians administer thrombolysis appropriately with this change in setting.

\section{METHODS \\ Setting}

Crosshouse Hospital is a 450 bed district general hospital in a mixed urban and rural area (population served about 240000 ) with a mortality from coronary heart disease among the highest in Scotland. ${ }^{25}$ Primary angioplasty and rescue angioplasty are not currently available in this hospital. During the study period, resident middle grade emergency medicine staff were available 24 hours a day.

\section{Inclusion and exclusion criteria}

Inclusion criteria for this study were all patients admitted to CCU with either a STEMI and/or thrombolysis (either given

Abbreviations: STEMI, ST elevation myocardial infarction; DTN, door to needle time; CCU, coronary care unit; ED, emergency department; CTN, call to needle time 
Table 1 Age, sex, and type of STEMI data for the prechange group and the post-change group

\begin{tabular}{llll}
\hline & Pre-change & Post-change & $\mathbf{p}$ \\
\hline Number of patients & 632 & 654 & - \\
Mean age (y) (SD) & $65.5(11.9)$ & $65.4(13.1)$ & $0.9^{*}$ \\
M: $F$ ratio & $1.6: 1$ & $2.0: 1$ & $0.037 \dagger$ \\
Streptokinase: rPA ratio & $7.9: 1$ & $2.5: 1$ & $<0.001 \dagger$ \\
Inferior STEMI & 257 & 269 & $0.9 \dagger$ \\
Anterior STEMl & 209 & 197 & $0.4 \dagger$ \\
Lateral Ml & 22 & 37 & $0.075 \dagger$ \\
Non- $Q$ wave Ml & 67 & 62 & $0.5 \dagger$ \\
Posterior STEMl & 4 & 4 & $1.0 \dagger$ \\
STEMl, site not specified & 73 & 85 & $0.5 \dagger$ \\
\hline *Two sample $t$ test; $\dagger \chi^{2}$ test. & & \\
\hline
\end{tabular}

or to be given, see below). Patients with clinical delays such as uncontrolled hypertension, uncertainty about thrombolysis decision, and obtaining consent were also included in the study.

Exclusion criteria were: inpatients who were transferred to CCU more than 24 hours after hospital admission; patients in whom the diagnosis of STEMI was a secondary event (unrelated to the original reason for admission); patients transferred to CCU from another hospital; patients who were thrombolysed with a final diagnosis of massive pulmonary embolism; patients admitted with intermittent cardiac chest pain with no initial diagnostic ECG changes.

\section{Clinical course and study intervention}

All patients are admitted through the ED and initial assessment is carried out by ED staff. Until 1 April 2000, thrombolytic therapy was always given in the CCU by the duty junior doctor on call for general medicine. After this date the primary site for the administration of thrombolysis was moved to the ED where the thrombolysis decision was made by the duty emergency department medical staff. ED medical and nursing staff were given a one hour lecture by a consultant cardiologist on the risks, benefits, and protocol for administration of thrombolytic agents for STEMI before the change in location for thrombolysis to the ED. This lecture is repeated every six months for each new intake of junior emergency medicine staff (medical and nursing).

The study prospectively compared the period from 1 April 1998 to 31 March 2000 (pre-change group) with the period from 1 April 2000 to 31 March 2002 (post-change group). The decision to administer thrombolysis was based on the presence of a clinical myocardial infarction and a diagnostic electrocardiograph ${ }^{23}$ and the absence of existing contraindications. Senior clinicians (duty consultant cardiologist or emergency medicine consultant) were consulted when there was any diagnostic uncertainty. There was no change to the protocol for treatment of STEMI over the course of the study.

An ECG was performed as soon as possible by an ECG technician or ED nurse when patients suspected of suffering from STEMI arrived in the ED with a target of within five minutes of arrival. All ECGs were scrutinised immediately by ED medical staff.

Intravenous reteplase was given in the following circumstances: patient less than 50 years of age, presenting within four hours of the onset of chest pain with an anterior STEMI; hypotensive patient (systolic blood pressure $<90 \mathrm{~mm} \mathrm{Hg}$ ); or if the patient had been given streptokinase previously. All other patients were given intravenous streptokinase. The dose of reteplase was 10 units intravenous bolus, repeated after 30 minutes. An intravenous bolus of 5000 IU unfractionated heparin was also given as per protocol, followed by an intravenous infusion of 1000 IU heparin per hour. The
Table 2 Comparison of door to needle times and ambulance call to needle times for each group

\begin{tabular}{lll}
\hline & $\begin{array}{l}\text { Door to needle } \\
(\mathbf{m i n})\end{array}$ & $\begin{array}{l}\text { Call to needle } \\
(\mathrm{min})\end{array}$ \\
\hline $\begin{array}{l}\text { Pre-change group } \\
\text { median (IQR) }\end{array}$ & $64(46-95)$ & $102(83-129)$ \\
$\begin{array}{l}\text { Post-change group } \\
\text { median (IQR) }\end{array}$ & $35(25-65)$ & $\begin{array}{l}77(59) \\
(\mathrm{n}=275)\end{array}$ \\
$\begin{array}{l}\text { Estimated difference } \\
(\mathrm{n}=323)\end{array}$ & $25(20$ to 29$)$ & $23(18$ to 28$)$ \\
(95\% Cl) & $<0.0001$ & $<0.0001$ \\
$\mathrm{p}^{*}$ & & \\
\hline *Mann-Whitney $U$ test. IQR, interquartile range.
\end{tabular}

dose of streptokinase was 1.5 million units by intravenous infusion over 60 minutes.

Patients were routinely kept in the ED for at least 15 minutes after administration of thrombolytic therapy and were then transferred to CCU with a nurse and doctor escort with defibrillator and resuscitation equipment.

Patients who had a convincing history but no diagnostic ECG were also admitted to CCU or the acute medical receiving ward as appropriate and treated for unstable angina/acute coronary syndrome. The ECG was repeated as felt clinically appropriate. Patients who were thought to have non-cardiac chest pain were assessed and admitted to the acute medical receiving ward or discharged from the ED as clinically indicated.

Patients in whom there was a clinical delay such as uncontrolled hypertension or diagnostic uncertainty could be transferred to CCU before thrombolysis at the discretion of the treating physician.

\section{Data collected}

Data were routinely collected prospectively on all those patients who were given thrombolytic therapy and on all patients admitted to CCU with suspected STEMI. Data from the period l April 1998 to 31 March 2002 were used for this study, giving rise to two distinct time periods, namely the pre-change group (intention to thrombolyse in CCU) from l April 1998 to 31 March 2000 and the post-change group (intention to thrombolyse in ED) from 1 April 2000 to 31 March 2002.

\section{Patients who were thrombolysed with a STEMI}

All patients with a discharge diagnosis of STEMI during the study period were analysed. Discharge diagnosis of STEMI was based on the original WHO criteria for the diagnosis of STEMI (that is, two of clinical history, ECG changes and cardiac enzyme rises). ${ }^{26}$ Troponin assays were not available at this centre during the entire study period. Data collection included age and sex; time of calling for an ambulance (where appropriate, defined as the "call time"); time of arrival in the ED (defined as the "door time"); mode of arrival in CCU (self referral to ED; ambulance service to ED; GP referral; transfer to CCU from inpatient ward); whether or not thrombolytic therapy was administered; if so, was it administered in the ED or CCU; time of start of thrombolytic therapy (ED or CCU, defined as the "needle time"); site of STEMI; final diagnosis; complications; length of stay in CCU; survival to CCU discharge or death.

Complications were defined as serious adverse events such as ventricular arrhythmia, haemorrhage, or cerebrovascular accident.

From these basic data, it was possible to calculate call to needle (where ambulance transport was used) and door to needle times. Times were measured to the nearest minute. The definitions used are consistent with those used in the MINAP audit in England. ${ }^{20}$ 
Table 3 Percentage of patients who meet national standards for emergency thrombolysis

\begin{tabular}{|c|c|c|c|c|}
\hline & Standard & Pre-change & Post-change & $\mathbf{p}^{*}$ \\
\hline $\begin{array}{l}\text { NSF } 2000^{28} \\
\text { CSBS } 2000^{32}\end{array}$ & $\mathrm{CTN}<60 \mathrm{~min}$ & $3.6 \%(10$ of 275$)$ & $24.9 \%$ (68 of 273 ) & $<0.001$ \\
\hline $\begin{array}{l}\text { NSF } 2002^{28} \\
\text { AHA } 1999^{33}\end{array}$ & $\mathrm{DTN}<30 \mathrm{~min}$ & $4.1 \%(13$ of 320$)$ & $38.7 \%(125$ of 323$)$ & $<0.001$ \\
\hline NSF $2003^{28}$ & $\mathrm{DTN}<20 \mathrm{~min}$ & $0.3 \%$ (1 of 320 ) & $13.0 \%$ (42 of 323 ) & $<0.001$ \\
\hline
\end{tabular}

\section{Patients who were not initially thrombolysed with a STEMI}

All those patients who were admitted to the acute medical ward and subsequently transferred to CCU for thrombolysis were subjected to case note review to identify distinct second events and to identify the possibility of initial misdiagnosis on ECG. As all in-patients are assessed by a consultant physician within 24 hours of admission, it was considered that any transfers to CCU more than 24 hours after admission were unlikely to have had a STEMI at ED presentation. These were classified as secondary events. A secondary event was defined as a case in which STEMI occurred in patients who were admitted without initial evidence of STEMI and had a separate episode of chest pain with a subsequent diagnosis of STEMI. Continuing pain with evolving ECG changes was not considered to be a secondary event.

\section{Patients who were thrombolysed but did not have a STEMI}

Some patients did not have a discharge diagnosis of STEMI but were given thrombolytic therapy in the ED or CCU by medical staff. To further assess the appropriateness of the decision to administer thrombolysis, two of the authors (JNA, a consultant cardiologist and ACMcG, a consultant in emergency medicine) individually reviewed the cases.

The clinical data available to medical staff at the time of making the thrombolysis decision were abstracted on to a standard form by another author (ARC) and presented to the two assessors with the admission ECG. The assessors were blinded as to where thrombolysis had been administered and which specialty had been responsible for the decision.

They were asked to state whether or not the symptoms given were consistent with STEMI; whether or not the ECG was consistent with STEMI; whether or not they would have admitted this patient to CCU; and whether or not they would have given thrombolytic therapy with the given history and ECG. These results were used to classify patients into two categories: "appropriate thrombolysis" and "inappropriate thrombolysis". If there was disagreement between the two assessors on any of the four questions, it was classified as "unable to decide".

In addition, the notes of 20 randomly selected patients who sustained a STEMI and received thrombolytic therapy were also abstracted in an identical manner to that described already. These forms were not identified separately to the assessors and were given to act as a control group.

\section{Statistical analysis}

Data were entered on to a Microsoft Excel spreadsheet initially and transferred on to Minitab (v13.30) for data analyses. Descriptive data are presented as means for normally distributed data and medians for non-parametric data. The two sample $t$ test was used to compare continuous normally distributed data. The Mann-Whitney $U$ test was used to compare non-parametric continuous data such as door to needle times. The $\chi^{2}$ test was used to compare categorical data. The $95 \%$ confidence intervals (CIs) are given where appropriate. Statistical significance was defined as $\mathrm{p}<0.05$.

\section{RESULTS}

A total of 1349 patients were admitted to CCU with a diagnosis of STEMI and/or had received thrombolytic therapy during the four year period. Of these, a total of 63 patients were excluded from further analysis. Of the excluded patients, 16 were transferred to CCU from the in-patient wards more than 24 hours after admission through the ED, 27 patients had a diagnosis of STEMI where the STEMI had clearly been a secondary event, 11 patients were transferred directly to CCU from outlying hospitals, and two patients had a diagnosis of massive pulmonary embolism and were given thrombolytic therapy. Seven patients were excluded who had an eventual discharge diagnosis of STEMI but were admitted to the acute medical receiving ward with intermittent cardiac chest pain but no initial diagnostic ECG changes. Four of these patients were in the pre-change group and three were in the post-change group.

These exclusions meant there were a total of 1286 patients available for analysis, 632 patients in the pre-change group and 654 patients in the post-change group.

\section{Patients who were thrombolysed with a STEMI}

The pre-change group contained 319 patients who received thrombolytic therapy in CCU. In addition, two patients from the pre-change group were thrombolysed in the ED because of pressures on CCU beds. In the post-change group, of those who were thrombolysed, $73.5 \%$ (238 of 324 ) were given thrombolytic therapy in the ED and $26.5 \%$ (86 of 324) were given thrombolytic therapy in CCU. Patients in the postchange group who were given thrombolysis in CCU included those with delays for clinical reasons and one patient in whom the diagnosis of STEMI was missed in ED.

Both groups were well matched. Table 1 gives the age, sex, and type of STEMI data. There were more men in the postchange group, and significantly more reteplase was used in the post-change group compared with the pre-change group. There were no other significant differences between the baseline characteristics of the two groups.

The proportion of patients with a discharge diagnosis of STEMI who were given thrombolytic therapy was 50.8\% (321 of 632) in the pre-change group and $49.5 \%$ (324 of 654) in the post-change group $(\mathrm{p}=0.8)$. This gives rise to an overall thrombolysis rate of $50.2 \%$ (645 of 1286) for the entire MI cohort.

The median DTN time was 64 minutes for the pre-change group and 35 minutes for the post-change group (point estimate for difference 25 minutes, $95 \%$ CI 20 to 29 minutes; $\mathrm{p}<0.0001)$. This, and details of call to needle (CTN) time, are given in table 2. Table 3 gives the percentage of patients in each group who achieved various national standards.

Note that the DTN time was not available for one patient in each group. There were 46 patients with missing CTN times for the pre-change group and 51 patients with missing CTN times in the post-change group. These were mostly patients 
who self presented to the ED with symptoms of a STEMI rather than calling for help.

The number of patients developing complications was similar for each group (pre-change, 32 patients; post-change, 22 patients; $\mathrm{p}=0.1$ ).

CCU mortality was 9.7\% (61 of 632) for the pre-change group and $8.3 \%$ (54 of 654) for the post-change group $(p=0.4)$ and median duration of stay in CCU was four days for the pre-change group and four days for the post-change group $(\mathrm{p}=0.6)$.

\section{Patients who were not initially thrombolysed with a STEMI}

Over the four year period, four of $608(0.7 \%)$ patients that received thrombolysis and had a final diagnosis of STEMI had been inappropriately directed to acute medical receiving unit by ED staff when there was clear clinical and ECG evidence of STEMI at initial presentation.

\section{Patients who were thrombolysed but did not have a STEMI}

From all 1286 patients, a total of 37 patients were in this subset. It was not possible to locate two sets of case notes; therefore 35 case notes were available for analysis. Agreement on the decision to administer or withhold thrombolysis was reached between the blinded cardiologist and emergency physician in 24 cases $(69 \%)$. In 15 of these 24 cases $(62 \%)$, thrombolytic therapy had been given "appropriately" on the basis of the presenting symptoms and initial ECG. In nine cases $(38 \%)$, thrombolytic therapy was judged to have been given "inappropriately". Of these nine cases, seven received thrombolysis in CCU and two received thrombolysis in the ED, with no significant difference between the groups $(p=0.36)$. In the remaining 11 cases $(31 \%)$, agreement was not reached.

The case notes of a further 20 patients who had a discharge diagnosis of STEMI and who received thrombolytic therapy were analysed in the same way as described above to identify any differences between the expert assessors. Agreement on thrombolysis was reached in $90 \%$ (18 of 20) of cases. Each assessor would have withheld thrombolysis from one eligible patient in this group, giving the specialists a 95\% "correct" thrombolysis rate.

\section{DISCUSSION}

This study has shown a significant improvement in DTN and CTN times when the primary site of hospital thrombolysis is moved from CCU to the ED. Importantly, this study involved all patients with STEMI presenting to a district general hospital, not just patients with a STEMI eligible for thrombolysis at presentation.

The two patient groups were broadly similar. Reteplase was used significantly more in the post-change group. This may reflect an increased number of patients presenting with a second STEMI in whom repeat streptokinase is contraindicated. It may also reflect a changing approach to thrombolysis and a move to increased use of recombinant thrombolysis through time.

We have also shown that existing ED staff, with senior emergency medicine and cardiology support, are able to make the sometimes difficult decision as to whether thrombolysis is appropriate for more complex patients. Although there was a significantly higher number of patients thrombolysed in the post-change group without a final diagnosis of STEMI, the retrospective blinded analysis by two experienced clinicians judged that in only two cases in the post-change group was this decision "inappropriate". Seven patients were deemed to have received "inappropriate" thrombolysis in the prechange group.
The difficulties in making the decision to administer thrombolysis in some cases are highlighted by the fact that two experienced clinicians were unable to agree in 11 of 35 patients without a final diagnosis of STEMI. Although they did not have the benefit of clinical examination, disagreement remained despite identical blinded information. This reflects the significant minority of patients for whom the decision to give thrombolytic therapy is difficult.

Similarly we have shown ED staff rarely miss the opportunity to provide thrombolytic therapy to patients presenting with clinical and ECG evidence of STEMI, with only $0.7 \%$ of patients being inappropriately admitted to the acute medical assessment unit with a diagnosis of unstable angina or acute coronary syndrome. The small number involved (four patients) precludes any further comment on the impact of the change in primary site of thrombolysis.

Even in a well organised ED with motivated staff, the median DTN remained at 35 minutes. Although the delays associated with transfer and duplication of assessment ${ }^{11} 12172^{23}$ have been removed, there remain some areas for potential improvement. An aging population with complex medical problems requires careful assessment and sometimes mandates comparison of the presenting ECG with previous ECGs, which can be a cause of delay. Electronic access to patient records or previous ECGs, or both, may help to reduce this delay. Nurse initiated ECG at triage for patients with chest pain and increased use of ambulance pre-alerting of patients with clinical STEMI may also help to further reduce DTN times.

Alternative solutions such as "fast track" systems ${ }^{13}$ have been criticised as they do not permit the provision of thrombolytic therapy by the first health professional capable of administering it (in most of the cases, ED staff or cardiology staff working in the ED).

Studies advocating direct access to the CCU have shown impressive results, ${ }^{14}$ but they cannot deal with the significant proportion of patients with STEMI who self present to the ED and would have no direct access to CCU. Current estimates within our hospital indicate that only around 25\% to $30 \%$ of CCU admissions are first assessed by general practitioners. Triage protocols by NHS 24 (NHS Direct in England and Wales) advise direct admission to hospital by emergency ambulance for cardiac sounding chest pain without the involvement of the general practitioner.

Prehospital thrombolysis has been suggested as a further way to improve CTN times. ${ }^{27}$ This will require time and resources for further training and equipment for paramedic staff. Some models also require remote hospital based medical support for thrombolysis decisions. Prehospital thrombolysis may be of benefit in rural or semi-rural environments. In the UK, a large proportion of the population live in an urban environment with quick (<30 minutes) access to an emergency department. The benefits of prehospital thrombolysis for urban patients remain unproven and it is possible that ED thrombolysis after rapid transport may be a more effective option.

Our results compare favourably with a similar study from Australasia. ${ }^{24}$ Despite this, we had difficulty in complying with the NSF $2002^{28}$ guidelines for thrombolysis times, which recommended that $75 \%$ of eligible patients have thrombolysis administered within 30 minutes of arrival in the ED. A recent Department of Health review of the National Service Framework for Coronary Heart Disease commented that there may be little clinical benefit reducing the DTN target to 20 minutes but possibly an increase in clinical risk associated with this. ${ }^{29}$ Our experience suggests that raising the standard to this very short time may impair clinical safety with little evidence of clinical benefit, especially given some of the diagnostic difficulties involved. 
It has been suggested that the future of early treatment of STEMI is primary angioplasty. ${ }^{5031}$ Cardiology services in most areas of the UK, Australasia, and outwith major centres in the US, are currently unable to provide this on a routine basis and are unlikely to be able to for some considerable time. $^{31}$ A more realistic alternative may be early ED thrombolysis and increased use of rescue thrombolysis or rescue angioplasty for failed reperfusion.

In conclusion, this study shows that moving the primary site of hospital thrombolysis from CCU to ED significantly improves DTN times and that ED staff can administer thrombolysis appropriately.

\section{Limitations}

Our study is a prospective observational study that monitored a change in clinical practice and not a randomised controlled trial. It is possible that the dataset may be incomplete. Patients admitted to our hospital with a STEMI may not have passed through CCU. However, all patients receiving thrombolysis in our hospital have to be admitted to CCU, therefore any patients with STEMI not admitted to CCU, could not have received thrombolysis and are not directly relevant to the study objectives.

It is possible that some of the improvements witnessed may have been a result of the study process rather than an improvement in clinical care, the Hawthorne effect.

The increased use of reteplase in the post change group may have led to an improvement in DTN times in itself as reteplase is quicker to prepare than streptokinase.

Ongoing staff education and changes in attitudes outwith the remit of the study over the course of the study may also have led to improved DTN times and improved appropriateness of thrombolytic therapy delivery.

\section{ACKNOWLEDGEMENTS}

We would like to thank all the staff of the coronary care unit and the emergency department for their support of this project and their continuing desire to improve the delivery of emergency cardiac care. We particularly thank those staff that entered data prospectively on to the database in CCU and those in the ED who collected data for the project. We also thank Dr David O'Neill, consultant cardiologist, and Ms Denise Brown, clinical audit coordinator, for their support of this project.

\section{Authors' affiliations}

A R Corfield, A C McGuffie, Accident and Emergency Department,

Crosshouse Hospital, Kilmarnock, UK

C A Graham, Accident and Emergency Department, Southern General Hospital, Glasgow, UK

J N Adams, Cardiology Department, Crosshouse Hospital

I Booth, Coronary Care Unit, Crosshouse Hospital

Funding: none.

Conflicts of interest: none declared.

\section{REFERENCES}

1 The GUSTO investigators. An international randomized trial comparing four thrombolytic strategies for ST elevation myocardial infarction. N Engl J Med 1993;329:673-82.

2 Fibrinolytic Therapy Trialists' (FTT) Collaborative Group. Indications for fibrinolytic therapy in suspected ST elevation myocardial infarction: collaborative overview of early mortality and major morbidity results from all randomised trials of more than 1000 patients. Lancet 1994;343:311-22.

3 Koren G, Weiss AT, Hasin Y, et al. Prevention of myocardial damage in acute myocardial ischemia by early treatment with intravenous streptokinase. N Engl J Med 1985;313:1384-9.

4 Lau J, Antman EM, Jimenez-Silva J, et al. Cumulative meta-analysis of therapeutic trials for myocardial infarction. N Engl J Med 1992;327:248-54 5 De Belder MA, Hall JA. Infarct angioplasty. Heart 1999;82:399-401.

6 Boersma E, Maas AC, Deckers JW, et al. Early thrombolytic treatment in ST elevation myocardial infarction: reappraisal of the golden hour. Lancet 1996;348:771-5
7 Boisjolie CR, Sharkey SW, Cannon CP, et al. Impact of a thrombolysis research trial on time to treatment for ST elevation myocardial infarction in the emergency department. Am J Cardiol 1995;76:396-8.

8 Newby LK, Rutsch WR, Califf RM, et al. Time from symptom onset to treatment and outcomes after thrombolytic therapy. GUSTO-1 Investigators. J Am Coll Cardiol 1996;27:1646-55.

9 Selker HP, Griffith JL, Beshansky JR, et al. Patient-specific predictions of outcomes in myocardial infarction for real-time emergency use: a thrombolytic predictive instrument. Ann Intern Med 1997; 127:538-56.

10 Topol EJ, Ohman EM, Armstrong PW, et al. Survival outcomes 1 year after reperfusion therapy with either alteplase or reteplase for ST elevation myocardial infarction: results from the global utilization of streptokinase and t-PA for occluded coronary arteries (GUSTO) III Trial. Circulation 2000;102:1761-5.

11 Birkhead JS. Time delays in provision of thrombolytic treatment in six district hospitals. Joint Audit Committee of the British Cardiac Society and a Cardiology Committee of Royal College of Physicians of London. BMJ 1992;305:445-8

12 Gonzalez ER, Jones LA, Ornato JP, et al. Hospital delays and problems with thrombolytic administration in patients receiving thrombolytic therapy: a multicenter prospective assessment. Virginia Thrombolytic Study Group. Ann Emerg Med 1992;21:1215-21.

13 Pell AC, Miller HC, Robertson CE, et al. Effect of "fast track" admission for ST elevation myocardial infarction on delay to thrombolysis. BMJ 1992;304:83-7.

14 Prasad N, Wright A, Hogg KJ, et al. Direct admission to the coronary care unit by the ambulance service for patients with suspected myocardial infarction. Heart 1997;78:462-4.

15 Qasim A, Malpass K, O'Gorman DJ, Heber ME. Safety and efficacy of nurse initiated thrombolysis in patients with ST elevation myocardial infarction. BMJ 2002;324:1328-31.

16 Sandler DA. Paramedic direct admission of heart-attack patients to a coronary-care unit. Lancet 1998;352:1198.

17 Sharkey SW, Bruneete DD, Ruiz E, et al. An analysis of time delays preceding thrombolysis for ST elevation myocardial infarction. JAMA 1989;262:3171-4.

18 Somauroo JD, McCarten P, Appleton B, et al. Effectiveness of a 'thrombolysis nurse' in shortening delay to thrombolysis in ST elevation myocardial infarction. J R Coll Physicians Lond 1999;33:46-50.

19 Wilmshurst P, Purchase A, Webb C, et al. Improving door to needle times with nurse initiated thrombolysis. Heart 2000;84:262-6.

20 Royal College of Physicians of London. Myocardial infarction national audit project. London: Royal College of Physicians of London, 2004. http:// www.rcplondon.ac.uk/pubs/books/minap/index.htm (accessed 14 Jan 2004).

21 Bryant M, Kelly AM. "Point of entry" treatment gives best time to thrombolysis for ST elevation myocardial infarction. Australian Health Review $2001 ; 24: 157-60$

22 Chan WK, Lam KN, Lau FL, et al. Starting thrombolytic therapy for patients with ST elevation myocardial infarction in accident and Emergency department: from implementation to evaluation. Chin Med J 1998;111:291-4.

23 Edhouse JA, Sakr M, Wardrope J, et al. Thrombolysis in ST elevation myocardial infarction: the safety and efficiency of treatment in the accident and emergency department. J Accid Emerg Med 1999;16:325-30.

24 Hourigan CT, Mountain D, Langton PE, et al. Changing the site of delivery of thrombolytic treatment for ST elevation myocardial infarction from the coronary care unit to the emergency department greatly reduces door to needle time. Heart 2000;84:157-6.

25 Scottish Executive. Health in Scotland 2002. Edinburgh: Scottish Executive, 2002. http://www.scotland.gov.uk/library5/health/his02-06.asp (accessed 12 Sep 2003).

26 Tunstall-Pedoe H, Kuulasmaa K, Amouyel P, et al. Myocardial infarction and coronary deaths in the World Health Organization MONICA Project. Registration procedures, event rates, and case-fatality rates in 38 populations from 21 countries in four continents. Circulation 1994;90:583-612.

27 Department of Health. National service framework for coronary heart disease. London: HMSO, 2000. 28-35. http://www.doh.gov.uk/pdfs/ chdnsf.pdf (accessed 12 Sep 2003)

28 Department of Health. Review of early thrombolysis. London: Department of Health, 2003. http://www.doh.gov.uk/heart/thrombolysis/review/ thrombolysis 3.pdf (accessed 12 Sep 2003).

29 Pedley DK, Bissett K, Connolly EM, et al. Prospective observational cohort study of time saved by prehospital thrombolysis for ST elevation myocardial infarction delivered by paramedics. BMJ 2003;327:22-6.

30 Andersen HR, Nielsen TT, Rasmussen K, et al. A comparison of coronary angioplasty with fibrinolytic therapy in ST elevation myocardial infarction. N Engl J Med 2003;349:733-42.

31 Jacobs AK. Primary Angioplasty for ST elevation myocardial infarction-ls it worth the wait? N Engl J Med 2003;349:798-800.

32 Clinical Standards Board for Scotland. Secondary prevention following ST elevation myocardial infarction. Edinburgh: Clinical Standards, 2000. 29-32. http://www.clinicalstandards.org/pdf/finalstand/Chd.pdf (accessed 12 Sep 2003).

33 American College of Cardiology/American Heart Association. Guidelines for the management of patients with ST elevation myocardial infarction. Dallas, TX: AHA, table 4, 1999. http://www.americanheart.org/ presenter.jhtml? identifier $=1824$ (accessed 12 Sep, 2003 